\title{
Developing a Reduced Gravity Testbed for the Nanoparticle Field Extraction Thruster
}

\author{
Brittany D. Drenkow ${ }^{*}$, Thomas M. Liu ${ }^{\dagger}$, John L. Bell ${ }^{\ddagger}$, Mike X. Huang ${ }^{\S}$, Shane P. Moore ${ }^{\mathbb{I}}$, Joshua R. Robinson", \\ Vritika Singh $^{* *}$, Rachel E. Trabert ${ }^{\dagger \dagger}$, Brian E. Gilchrist ${ }^{* \neq}$, and Alec D. Gallimore ${ }^{\S \S}$ \\ University of Michigan, Ann Arbor, MI, 48109
}

\begin{abstract}
The Nanoparticle Field Extraction Thruster (NanoFET) is an electric propulsion device under development at the University of Michigan to electrostatically charge and accelerate micro- and nano-particle propellant. During the 2008-2009 academic year, undergraduates comprising the Zero-g ElectroStatic Thruster Testbed team within the Student Space Systems Fabrication Laboratory completed a full design-build-test-fly cycle to develop the first NanoFET prototype with a piezoelectric-based feed system and to implement a thruster testbed for future microgravity flights. Reduced gravity testing was conducted via NASA's Reduced Gravity Student Flight Opportunities Program based at NASA Johnson Space Center. During the course of the project, the team promoted student growth and future sustainability. The team's organization, work completed, and educational benefits are presented.
\end{abstract}

\section{Introduction}

$\mathrm{E}$ lectric propulsion, unlike chemical rockets, utilizes electric and magnetic forces to create thrust and provide efficient propellant usage for deep space missions and orbital maneuvering. The Nanoparticle Field Extraction Thruster (NanoFET) is an electric propulsion device for small spacecraft under development at the University of Michigan. NanoFET electrostatically charges and accelerates solid micro- and nano-particles to produce thrust. The goal of NanoFET is to leverage a single, flexible electric propulsion thruster to enhance and enable a broad range of missions. ${ }^{1}$

ZESTT (Zero-g ElectroStatic Thruster Testbed) is an undergraduate team affiliated with the University of Michigan's Student Space Systems Fabrication Laboratory (S3FL). Unlike previous S3FL reduced gravity experiments that worked with a liquid-based NanoFET configuration ${ }^{2}$, ZESTT focused on testing a dry propellant configuration of NanoFET through NASA's Reduced Gravity Student Flight Opportunities Program based at Johnson Space Center. During 2008-2009, the team developed a NanoFET prototype and a vacuum testbed usable in both terrestrial and microgravity environments to better understand NanoFET's operational parameters and design drivers.

S3FL is a student-run laboratory dedicated to providing undergraduate students with hands-on research experience through real-world, multidisciplinary space systems projects. ${ }^{3}$ The design-build-test-fly philosophy is central to S3FL projects requiring students with diverse skill sets from a variety of disciplines. The organization is overseen by an Executive Committee (Excom) who organizes, advises, and assesses the progress of each project. Teams are divided into smaller subsystem groups focused on specific aspects such as the structural or electrical

\footnotetext{
* Undergraduate Student, Aerospace Engineering, bdrenkow@ umich.edu, AIAA Student Member

† Graduate Student, Aerospace Engineering, liutm@umich.edu, AIAA Student Member

* Undergraduate Student, Electrical Engineering, johnlb@umich.edu

${ }^{\S}$ Undergraduate Student, Electrical Engineering, huxuli@umich.edu

"Il Undergraduate Student, Aerospace Engineering, shanemo@umich.edu

\# Undergraduate Student, Aerospace Engineering, joshrob@umich.edu

** Undergraduate Student, Aerospace Engineering, singhv@umich.edu

† Undergraduate Student, Aerospace Engineering, raetr@umich.edu

Professor, Electrical Engineering \& Space Systems, gilchrst@umich.edu, AIAA Associate Fellow

$\S$ Arthur F. Thurnau Professor, Aerospace Engineering \& Applied Physics, rasta@umich.edu, AIAA Associate Fellow
} 
systems. Students start in the lab as subsystem team members and, depending on their technical and leadership skills acquired over time, are able to work their way up the project hierarchy. Capable and dedicated team leaders are invited to become Excom members to oversee and direct laboratory activities. Involvement in S3FL enhances technical skills learned in the classroom and provides students with valuable real-world project experience.

Team ZESTT positively impacts not only the development of the future technical workforce but also enhances space technology development. By allowing NanoFET prototypes to be tested in "space-like" environments (i.e., both vacuum and microgravity), ZESTT provides an opportunity to increase the NanoFET concept's Technology Readiness Level (TRL) ${ }^{4}$, thus raising confidence in the technology's growing maturity. In addition, reduced gravity demonstrations like ZESTT help qualify NanoFET systems for use in future missions onboard microgravity flights or small satellite platforms involving free-floating units whose dynamics can be controlled by NanoFET operations.

\section{ZESTT Objectives and Team Organization}

The ZESTT project was created to aid in NanoFET's technical development while providing an educational experience to undergraduate students. A set of objectives (technical and educational) provided overall direction for the project. The team's organization supplied the means for these objectives to be implemented.

\section{A. Objectives}

The technical objectives set the overarching requirements for advancing NanoFET and provided future sustainability of microgravity experiments. These objectives were as follows:

1) Prototype a dry, piezoelectric-based feed system using microparticles.

2) Demonstrate the feed system's feasibility under vacuum conditions in terrestrial and microgravity environments.

3) Determine the feed system's throughput, emission uniformity, and efficiency for various throttling conditions.

4) Validate NanoFET performance models for particle charging, liftoff, and acceleration.

5) Implement a thruster testbed for future microgravity experiments.

The development of a prototype piezoelectric-based propellant feed system for NanoFET lies at the heart of the ZESTT project. As part of demonstrating the feasibility of this system, the prototype needed to undergo ground testing followed by a reduced gravity flight demonstration. In preparation for ZESTT's microgravity flight, a thruster testbed was created to provide future sustainability of NanoFET testing. From these objectives, team members formulated specific requirements to drive the design process.

While ZESTT is a technically-driven project, several educational objectives were derived from the team's involvement with S3FL to provide a challenging learning environment for each student, promote outreach activities, and compile documentation as a reference for future projects. The educational objectives for the ZESTT project were as follows:

6) Implement S3FL's design-build-test-fly methodology and track student development.

7) Provide "beyond the classroom learning" opportunities to engage pre-college students.

8) Compile standard project policies, procedures, and lessons learned for future S3FL teams.

\section{B. Team Organization}

In order to accomplish the technical and educational objectives set forth, the team adopted the hierarchy shown in Figure 1. ZESTT was divided primarily into five specialized subsystem teams: science, structures/mechanisms, electrical/command and data handling $(\mathrm{CDH})$, business, and outreach. Each team member was part of one or more of these specialized subsystems. The science team was primarily in charge of all NanoFET prototype testing while the structures/mechanisms team designed and fabricated the prototypes and supporting experiment chassis. The electrical/CDH team focused on all electrical and control systems, including a custom-built ammeter and LabVIEW automation code. The business team was created to handle all fundraising, publicity, and budget tracking for ZESTT. This subsystem developed a sponsorship package, contacted industry, and managed the team's internal business affairs. The outreach team was responsible for inspiring K-12 students in the areas of science and engineering. 


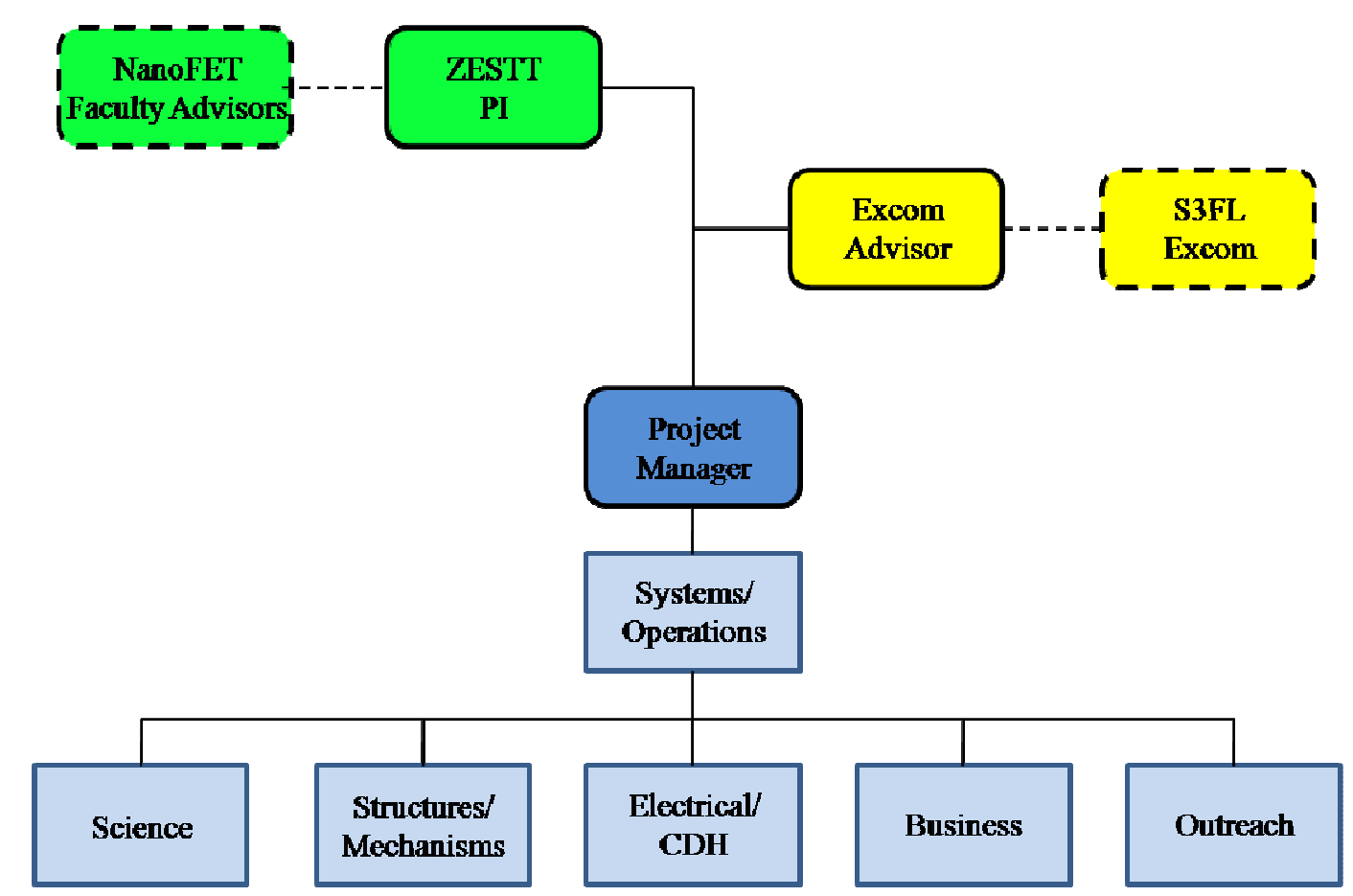

Figure 1. ZESTT Team Hierarchy. Each of the five focused subsystem teams was led by their own lead that together made up the Systems/Operations team.

All subsystems were led by a subsystem lead that, in conjunction with other subsystem leads, comprised the systems/operations team. The systems/operations team was overseen by the project manager who was advised by the principle investigator and S3FL Excom. In such a fast-paced technical project, communication between team members was especially important. Weekly meetings between the project manager and systems/operations team were held to allow each subsystem to update each other on their team's progress towards the overall objectives as well as to distribute action items. An online worksite with forums and resources was established as a means of sharing documents and information with the entire team. This site enhanced communication across subsystems, allowed for an organized means of compiling documentation, and aided the project's overall integration.

The ZESTT project's schedule was designed around four major milestones or reviews prior to flight. The first milestone was the Mission Definition Review (MDR). This review provided team members with the opportunity to communicate their preliminary baseline design to knowledgeable reviewers in exchange for feedback and advice. MDR was also a means to determine the objectives and driving requirements, identify high-risk items and mitigation strategies, and prepare the experiment proposal for submission to NASA's Reduced Gravity Student Flight Opportunities Program.

Following MDR and the proposal's selection, the team further developed the design and presented more specialized Preliminary Design Reviews (PDR) for separate aspects of the project including the NanoFET prototype, chassis and vacuum chamber, electrical/CDH systems, business, and outreach. These reviews were intended to establish a more detailed baseline design in preparation for the experiment's fabrication, identify additional high-risk items and mitigation strategies, and solicit expert advice. In April, a Go/No-Go demonstration was held to prove the experiment's functionality, and the Test Equipment Data Package (TEDP) was submitted to NASA to provide full documentation of the experiment's operation and potential safety concerns in preparation for microgravity flight testing. The team successfully passed all objectives of this review. Finally, once in Houston, the team had to pass a Test Readiness Review (TRR) conducted by NASA in order to fly. This review was intended to allow the test directors, flight operators, and other officials to identify any safety concerns and to ensure compliance with all regulations.

Each of these reviews presented the team with challenges. They learned how to work together under pressure to meet the deadlines, identify problems, and develop mitigation strategies early on. Technical communication skills were also enhanced through the development and execution of the presentations that involved a varied audience of faculty, peers, and professionals. 


\section{Experiment Design and Fabrication}

The NanoFET concept, schematically shown in Figure 2, requires that solid particles be electrostatically charged and accelerated to produce thrust. A charging electric field is generated via a potential bias between the particle sieve and the extraction gate electrode. Implementation of a piezoelectric-based feed system provides an inertial "kick-off" force at the particle sieve that allows for controlled particle liftoff and extraction. ${ }^{5}$

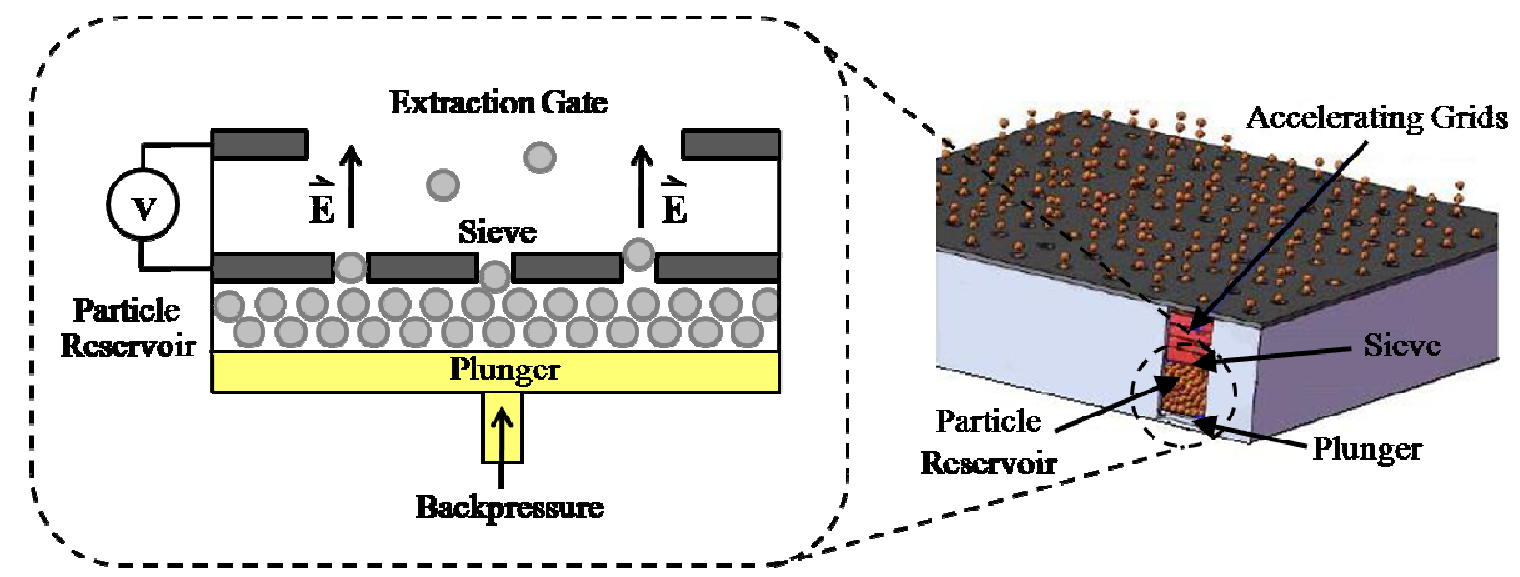

Figure 2. NanoFET concept with a cross-sectional view of the piezoelectric feed system. A plastic syringe was used as a particle reservoir contacting a nickel electroformed mesh sieve with a constant force spring providing the backpressure.

\section{A. M-1 NanoFET Prototype Development}

The NanoFET prototype, designed and built by the structures/mechanisms team, was the first generation prototype to use single micron-sized particles (1-10 $\mu \mathrm{m}$ silver-coated soda lime glass spheres) and was thereby designated the "M-1." The M-1 had a development life of approximately nine months. During this time, team members learned how to model their ideas using computer aided design (CAD) software such as SolidWorks followed by machining skills to create and assemble their product. The design of the M-1 went through several iterations, changing dramatically as student abilities improved and understanding of the concept increased. Figure 3 illustrates the prototype's progression through three main baseline iterations.

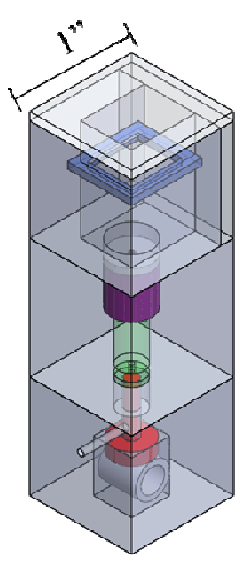

Oetober 2008

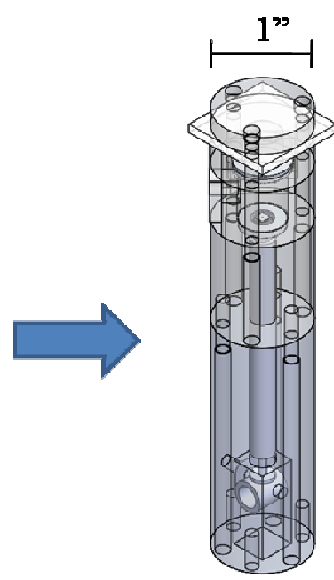

December 2008

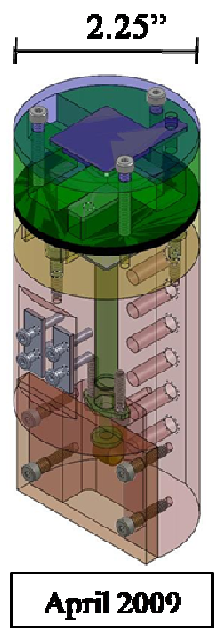

April 2009

Figure 3. Progressive designs of the M-1 prototype. Each design resulted in improved functionality.

The model on the left is the first iteration of the M-1 design. All of the main components (e.g., backpressure source, particle reservoir, charging electrodes, and anode) are present, but electrical connections, mounting schemes, and other interface details were not yet considered in this design.

The center diagram shows an effort to decrease the size of the M-1 and consider module connections/interfaces. This design featured "windows" or openings in the side of the M-1 near the top to allow for the particle tracking velocimetry (PTV) diagnostic (See Section III.C) to be used during ground testing. Components, such as the 
particle reservoir (chosen to be a plastic syringe), were also modeled to higher fidelity in the second phase. While NanoFET aims to reduce its size, later configurations of the M-1 featured a larger radius polycarbonate stock that was more forgiving to the student machinists.
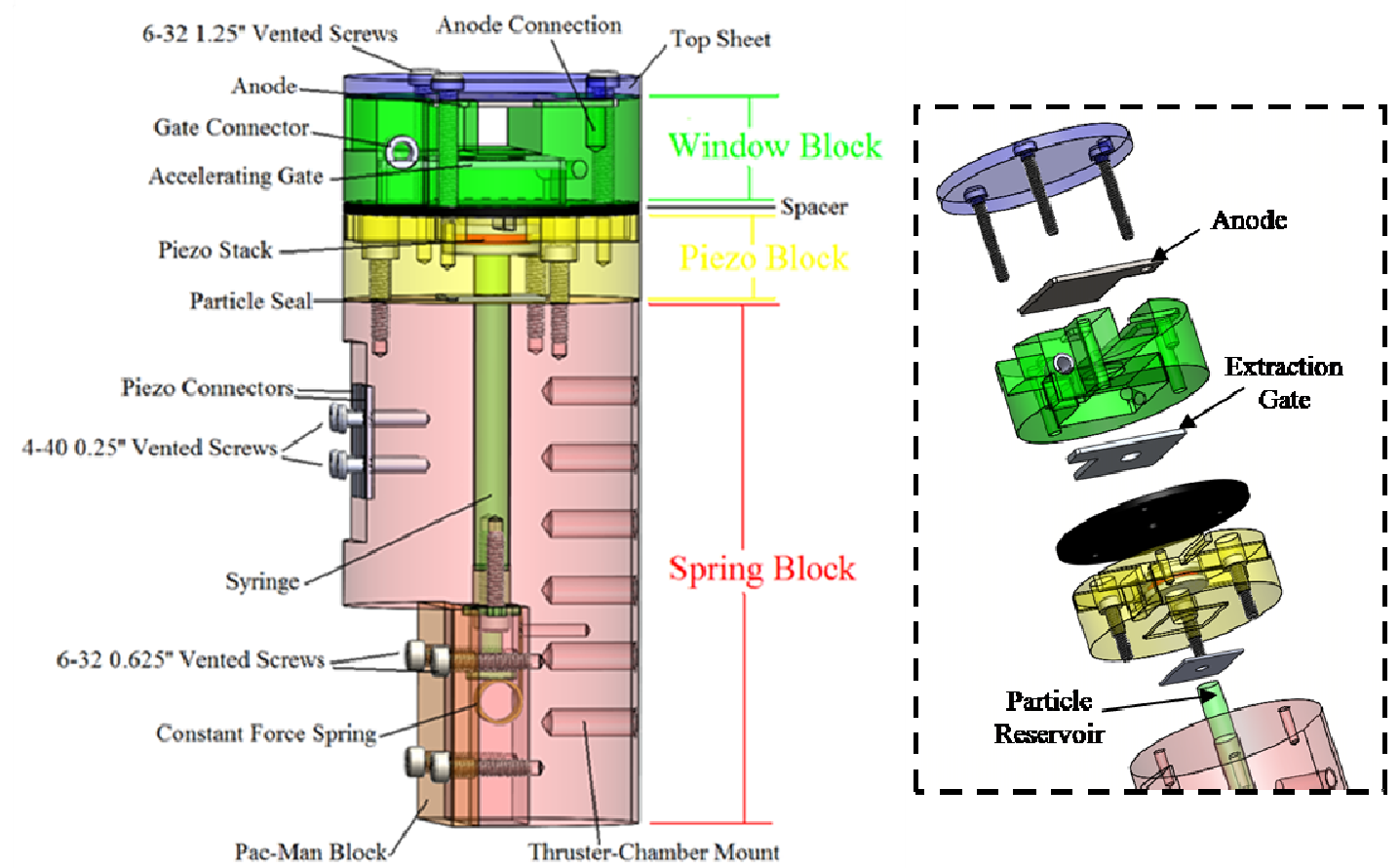

Figure 4. Modular M-1 flight prototype. Three modular blocks (window, piezo and spring blocks) allow all key parts to be removed and replaced if needed.

The diagram on the right of Figure 3, and shown in greater detail in Figure 4, is the flight configuration of the M1 prototype. This model was designed to be modular such that each section could be removed separately to allow easy accessibility and replacement of parts. The flight M-1 prototype consists of three main sections: window block, piezo block, and spring block. This design was the first to feature true electrical connections and includes more robust mounting schemes with fasteners such that the structures/mechanisms team could create full engineering drawings to be followed during fabrication. One of the most noticeable improvements in this design was the large portion milled out of the bottom of the M-1 in the spring block to aid in assembly practices by providing easier access to the constant force spring.

The M-1 prototype featured a piezoelectric feed system central to its operation. Three main components comprised the system, which included a piezoelectric assembly, particle reservoir (Becton, Dickinson and Company (BD) Medical 1-milliliter syringe), and backpressure source (Neg'ator constant force spring). The piezoelectric assembly is made up of a Noliac CMAR04 ring piezoelectric actuator, electroformed nickel sieve (10- $\mu \mathrm{m}$ apertures and $50-\mu \mathrm{m}$ pitch), and both ceramic (alumina) and stainless steel washers.

Great effort was put forth by the science team to understand the feed system's operational physics and the nuances affecting particle emission. Fundamentally, the feed system utilizes an applied vibration and backpressure to bring particles into contact with the sieve. As the particles pass through the sieve holes, which disperse particle clumps, they are charged and lifted off from the sieve where an applied electric field accelerates them to high speeds.

Due to the small size of the particles, they must overcome strong van der Waals forces in order to be extracted. As particles smaller than the millimeter size scale are used, the adhesion force becomes the dominant force in the system, thus requiring a strong electric field to extract the particle. ${ }^{6}$ It has previously been shown that microparticles can be manipulated by using piezoelectrics to supply inertial forces. ${ }^{7}$ As an oscillating piezoelectric's frequency is increased, the resulting inertial force is amplified. Therefore, as the vibration frequency is raised, the particle mass flow rate is hypothesized to increase as more layers of particles are kicked off the charging sieve and accelerated per unit time.

As particles are depleted, additional particles are forced into contact with the charging sieve by the constant force spring that pushes on the syringe plunger of the particle reservoir. Any particles that are extracted must pass 
through an extraction gate to impact the collection anode. Inefficiencies arise when some particles are diverted to the walls and gate rather than passing through towards the anode.

All of the M-1 components, with the exception of the stainless steel extraction gates, were fabricated in-house by the structures/mechanisms team at the Wilson Student Project Center on the University of Michigan's campus. Within this facility, the team could take advantage of a fully equipped machine shop (including basic tools, milling and lathing machines with CNC capabilities, and welding equipment), computer workstations, and individual space provided solely for sponsored student organizations such as S3FL. To operate the M-1 inside a vacuum chamber, special consideration was given to the components procured such that each had a low percentage (less than $1 \%$ ) of total mass loss (TML) in vacuum. A low TML reduces outgassing effects inside the vacuum chamber and therefore reduces the chances of contamination and undesirable electrical breakdown. Polycarbonate $(0.1 \% \mathrm{TML})$ was chosen for the M-1 prototype body, whose properties also allowed it to be easily machined while remaining highly durable without being brittle. Training on all machinery was conducted during the design phase, which team members then put to practice as they learned the process of fabricating the designs they created.

\section{B. Integrated System Design}

Testing in a reduced gravity environment required special considerations throughout the experiment's design and fabrication. The thruster testbed provided the framework for all terrestrial and reduced gravity testing. A schematic of the experiment tested onboard the NASA-contracted, Zero-G Corporation's Boeing 727 aircraft is shown in Figure 5. The testbed consisted of five main systems: NanoFET prototype, vacuum system, high voltage system, command and data system, and the chassis. Each system is unique to those studied in typical undergraduate courses and therefore required all team members to utilize the available resources (libraries, online publications, software, and personnel) to quickly become familiar with and accomplish their tasks.

Three M-1 prototypes were created for reduced gravity flight testing. The M-1 required its own piezoelectric control equipment to operate, including an Agilent 33220A arbitrary waveform generator to supply the piezoelectric drive signal that was then amplified using a Kepco BOP 100-1M bipolar operational power supply. A Pearson coil served to monitor the supplied current to the piezoelectric actuator, and a switching network transferred the drive signal to the desired prototype.

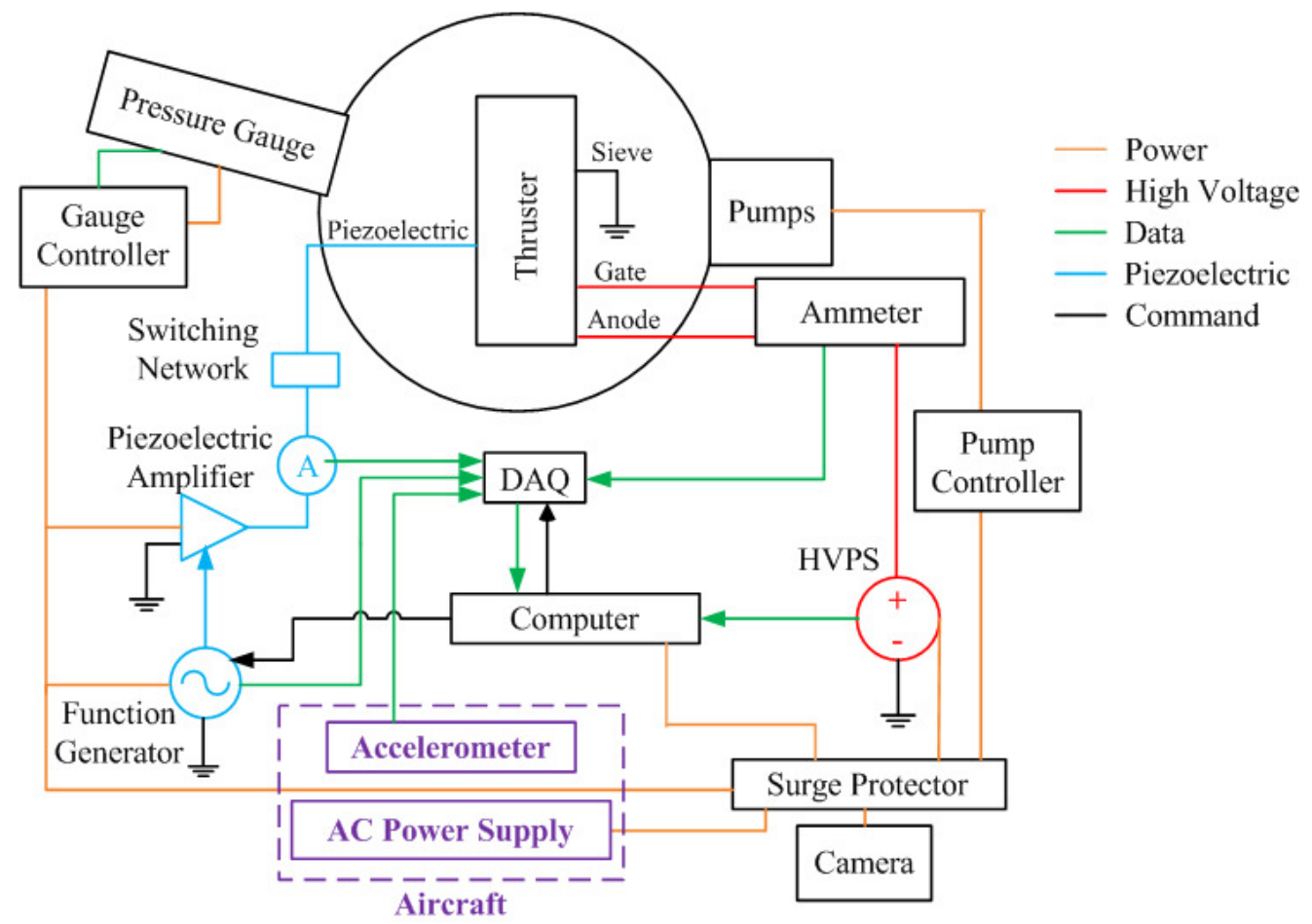

Figure 5. Microgravity experiment schematic. Three M-1 prototypes with supporting wiring were contained inside the vacuum chamber, the high voltage power supply contained three channels (one per M-1 prototype), and a custom-built ammeter attached to the high-voltage lines provided emission current measurements. 
The vacuum system was mainly the responsibility of the systems/operations team as it involved aspects from each of the other three technical subsystems (science, structures/mechanisms, and electrical/CDH). It consisted of a multi-port Kurt J. Lesker 12" stainless steel spherical vacuum chamber, a Varian dry scroll pump (IDP-3) and turbopump (Turbo V70-LP), and a Pfeiffer pressure gauge (PBR260). Placement of components on each port of the chamber required collaboration from students with different areas of expertise to take into account ergonomics,

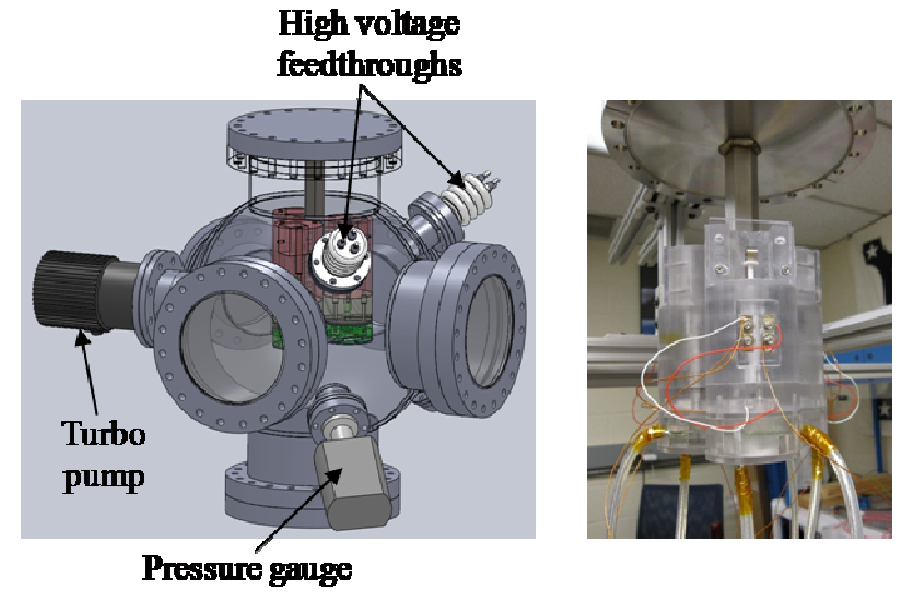

Figure 6. Mounting M-1 prototypes within the vacuum chamber. Three M-1 prototypes were mounted to a hexagonal rod welded to the top 8" flange for ease of making and removing electrical connections.

as indicating when the chamber pressure was beyond the Paschen curt to occur within the M-1 prototype. All unused flanges contained viewports rather than blanks to reduce the system's mass and allow increased visibility of the M-1 prototypes.

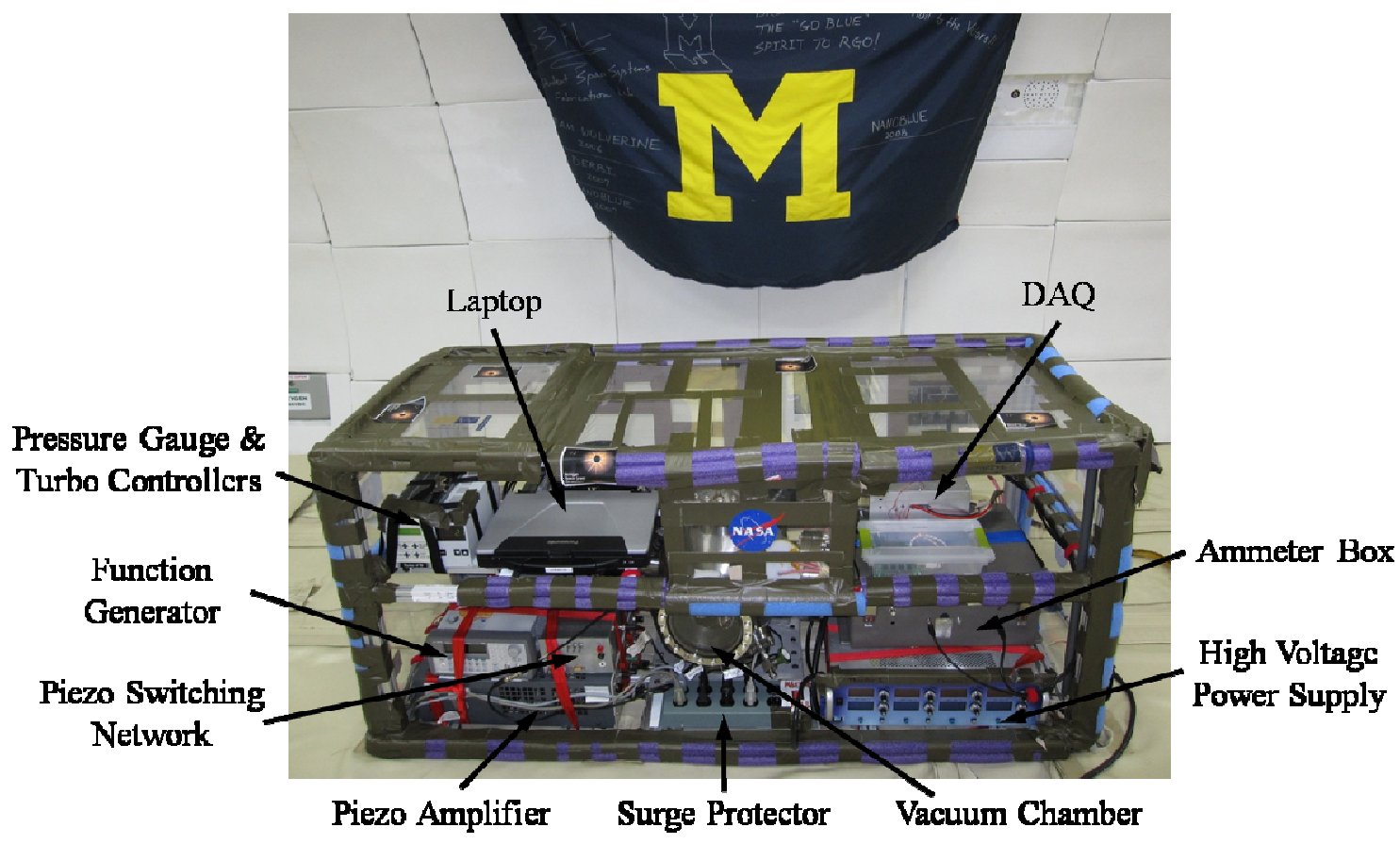

Figure 7. Microgravity flight experiment mounted on the Boeing 727 Zero-G aircraft. A dry scroll pump and turbopump were mounted behind the pictured equipment. Three M-1 prototypes were contained inside the vacuum chamber.

American Institute of Aeronautics and Astronautics 
In order to produce an electric field to charge and accelerate the microparticles within the M-1, high voltage was provided by an UltraVolt multichannel high-voltage power supply (HVPS). The electrical/CDH subsystem developed three custom-built ammeter circuits for emission current measurements on the high voltage lines.

The command and data system consisted of a Panasonic Toughbook 52 semi-rugged laptop enabling automation of the experiment via LabVIEW and a National Instruments USB-6218 data acquisition system (DAQ). The DAQ, having a total sampling rate of $250 \mathrm{kSamples} / \mathrm{s}$, acquired seven channels of data for the custom-built ammeter current, HVPS sense current and voltage, piezoelectric current and voltage, function generator output, and acceleration from the aircraft's onboard accelerometer. All data was sent and stored on the laptop computer for post-test analysis.

All of the equipment used in operating the M-1 prototypes in a vacuum environment was attached to a chassis housing, which provided the main structure that bolted to the aircraft as shown in Figure 7. The experiment chassis (59" long x 24" wide x 28 " tall) had strategically placed components for use by specific flyers during flight. Three flyers on each flight day were positioned next to each other in front of the chassis. Flyer 1 was primarily responsible for running the laptop, which contained the LabVIEW code (Section III.D) that automated the majority of the experiment. Flyer 2 observed the M-1 prototypes in the chamber and watched for possible arcs during operation. Flyer 3 operated the high voltage power supply and adjusted it accordingly if arcing occurred. Precaution was taken to protect the flyers and equipment during the 1.8-g period of the microgravity flight by covering the structure itself in foam padding and enclosing all sides in strong plastic shrink wrap.

Mass was a significant design driver for the experiment chassis. The team carefully kept a mass budget that tracked each component's weight to ensure everything was kept within NASA's limit (300 lbs $+50 \mathrm{lb}$ waiver).

The chassis utilized lightweight 6105-T5 aluminum beams from 80/20 Incorporated shown in Figure 8.

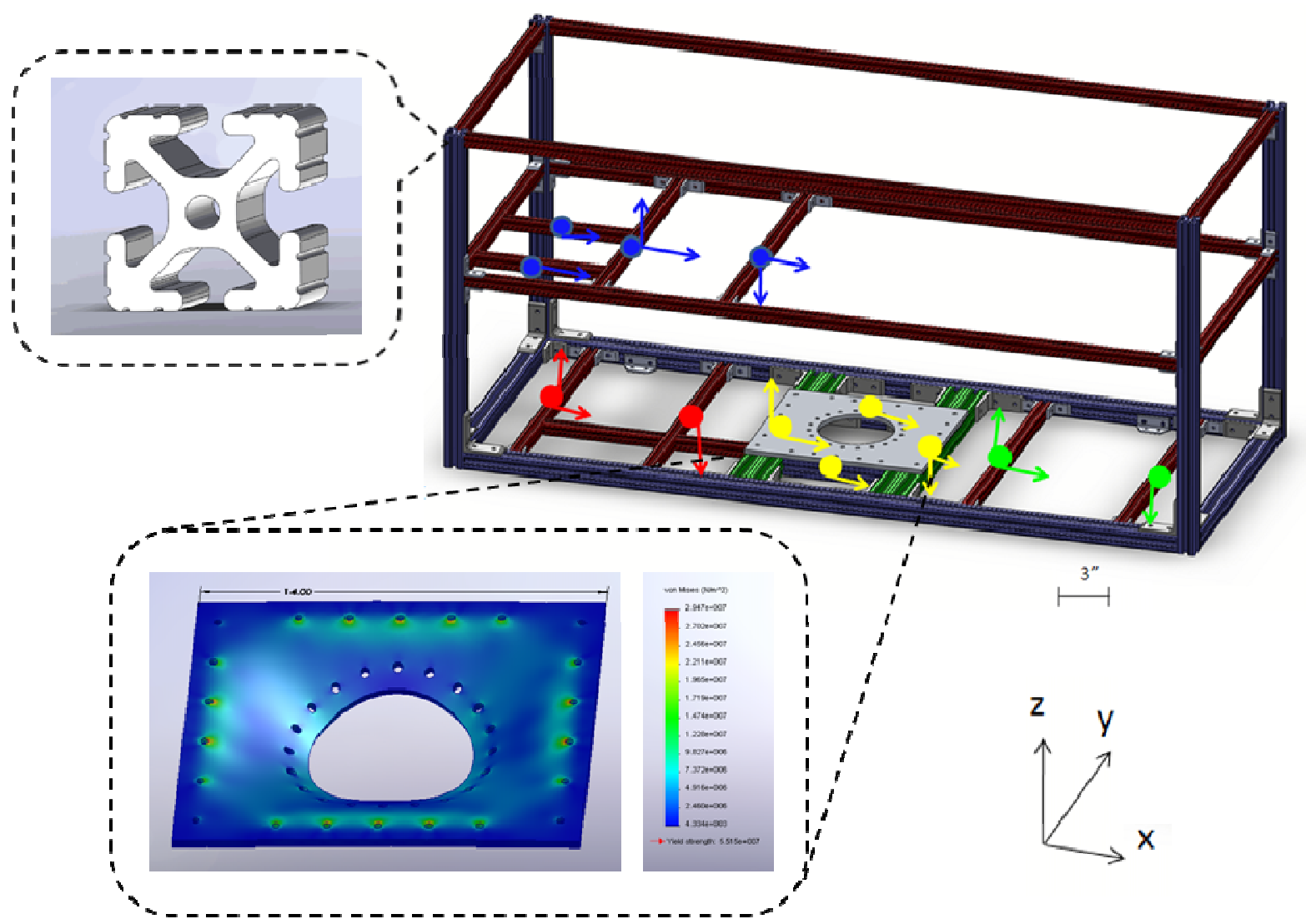

Figure 8. Experiment chassis. 80/20 beam cross-sections indicated as red for 1010, blue for 1515-Lite, and green for 1530-Lite. Point masses and resultant forces for 9-g acceleration are colored based on sets analyzed: Set 1 (red) - function generator, piezoelectric amplifier, and dry scroll pump; Set 2 (yellow) vacuum chamber, turbo pump, and power strip (yellow); Set 3 (green) - HVPS, ammeter, and DAQ; and Set 4 - laptop, pressure gauge, and turbo pump controllers. Example FEA for the vacuum chamber mounting plate is also shown. 
Structural verification techniques introduced in the classroom (a combination of hand calculations and finite element analysis (FEA) using NX I-DEAS V5.0) were applied to prove the chassis structure could survive under a variety of gravitational loading scenarios (9-g forward, 3-g aft, 6-g down, 2-g up, and 2-g lateral). Analysis and testing was also performed for impact and "kick loads" on both the chassis structure and the plastic wrap that enclosed the chassis. To ensure it would survive the worst case flight scenario, a minimum factor of safety of two was maintained. All requirements were successfully met with a minimum factor of safety of 2.86. Hand calculations were performed using Euler-Bernoulli beam theory and simple free body diagrams. Stress levels were overestimated using this method as loads were concentrated at the beam midpoints and at locations where beam failures were most likely to occur. All hand calculations agreed with the FEA results obtained to within $15 \%$.

\section{M-1 Diagnostics}

In addition to developing the M-1 prototype and supporting systems, the ZESTT team also developed unique diagnostic tools to characterize the prototypes. Three main diagnostic techniques were implemented: particle tracking velocimetry (PTV), ammeter data collection, and anode deposition analysis.

\section{Particle Tracking Velocimetry}

Typically, particle tracking velocimetry is a method utilized to characterize fluid dynamics by placing tracer particles in the fluid to be tracked and recorded. For the M-1, PTV is used as the primary ground testing diagnostic to determine the velocities of the extracted particles. The diagnostic utilizes a laser to illuminate the particles and a high-speed camera (Photron FASTCAM) to capture images. A custom MATLAB code was written to post-process the images and determine individual particle trajectories.

Placing the laser and FASTCAM orthogonal to each other, as shown in Figure 9, was found to maximize the interrogation zone that the FASTCAM could accurately image. Through experimentation, it was discovered that when the camera is placed at any other angle, the signal-to-noise ratio of the scattered light is lower, causing some particles to appear out of focus depending on their distance from the camera.

For the M-1 prototype testing, a helium-neon laser (632-nm wavelength) was used. A laser plane, achieved through a pair of small glass, argon-coated, plano-convex lenses from ThorLabs, Inc., maximized the area the particles could be traced. An approximate $1 \mathrm{~cm}$ square area served as the interrogation zone. The particles were on the micron size regime, thus allowing them to be large enough for the FASTCAM to visually observe the light being scattered while also being much smaller than the area within which they were being tracked. ${ }^{8}$

A custom post-processing program, developed in MATLAB, was created to determine the particle velocities. The code was designed such that it first reads in all of the images and converts them to a useable matrix from which the particles can then be

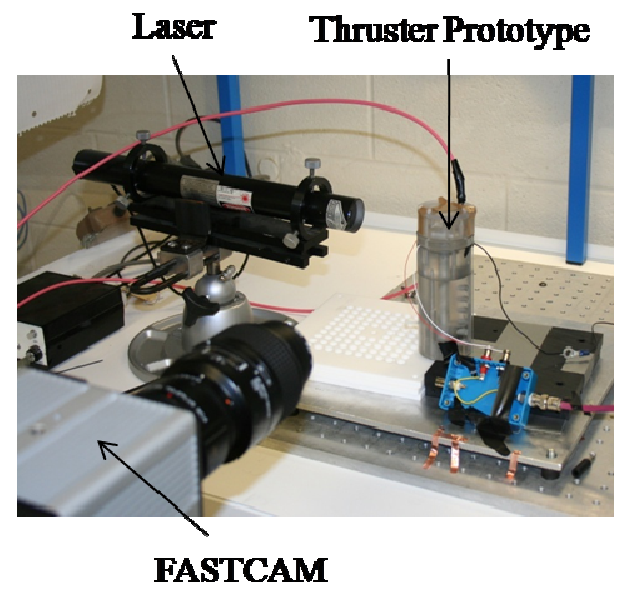

Figure 9. PTV setup. High-speed camera (FASTCAM) images particles within the M-1 illuminated by the laser plane created by a pair of cylindrical lenses (not shown). identified and tracked. A calibration is provided by the user that indicates the particle size and search area to be used such that stray particles and noise are removed. The particles are identified, and a database is created that records the particle's location and image number. In the successive images, the code searches for the same particles previously recorded and marks their new locations. Using the FASTCAM's known frame rate, the code is then able to calculate the velocity of each tracked particle based on the distances the particles traverse.

The PTV diagnostic provides a visual verification that the M-1 operates in addition to velocity information. As NanoFET moves towards smaller particles (nanometers), the output velocities will significantly increase. The resulting higher frame rates needed, which necessitates the use of more powerful lasers, become a greater challenge for smaller particles, especially since less light will be scattered by individual particles. Due to the expense of this system, the ZESTT team needed another means of characterizing the M-1 for reduced gravity testing. The solution was an ammeter. 


\section{Ammeter Design}

A custom ammeter was designed and built by the electrical/CDH subsystem as the main flight diagnostic used to measure particle emission as particles strike the collection anode. While the PTV diagnostic system offered simplicity, the team was challenged to develop a smaller, lighter method capable of being used in flight.

Several conventional devices were first investigated; however, none were found to have the ability of interfacing with the prototype to obtain the desired measurements. Hall effect sensors were considered but could not provide the nano-amp resolution that was expected from the microparticles emitted by the M-1 prototype. A Faraday cup, often used for similar purposes, was impractical since high voltage for the M-1 had to be applied on the collection end, rather than ground as is typical for Faraday cup collection. The team's custom ammeter diagnostic, similar to a Faraday cup, was therefore designed to measure current caused by particle charge transferred to the collection anode.

Several design iterations were conducted as electrical subsystem members learned how to isolate noise to measure nano-amp currents on a high voltage data line. Technicians and faculty were sought out to gain insight especially on high voltage operations and electrical noise. Circuitry knowledge taught in electrical engineering courses was put to use as the students prototyped each design, first on breadboards, before moving to PCB boards for the final configuration.

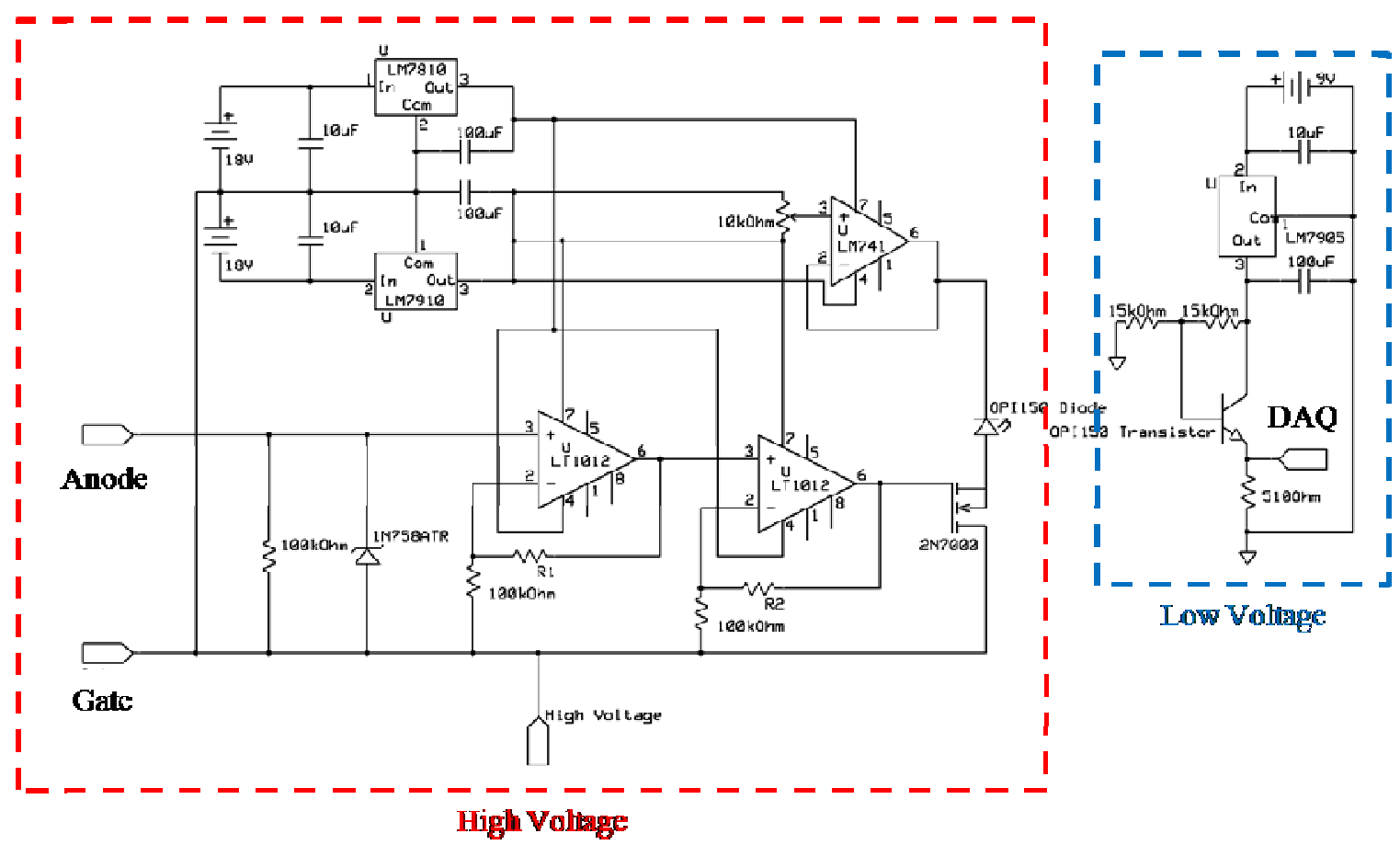

Figure 10. Custom ammeter for particle current measurements. The ammeter consists of two main sections: high voltage and low voltage. The two sections are separated by a light emitting diode (LED) and phototransistor to optically isolate high voltage signals from the low voltage data acquisition circuitry.

The flight ammeter was connected in series with the anode and acceleration gate of each M-1 prototype, and the differential current acquired due to particles was recorded. Current striking the anode was shunted over a $100-\mathrm{k} \Omega$ resistor (lower left side of Figure 10) and passed over two inverting amplifier stages. The output signal of the amplifier stages biased the gate of an N-channel metal-oxide-semiconductor (NMOS) transistor used to drive the opto-isolator LED on the right side of the high voltage stage. Since nonlinearities in the NMOS restricted its input signal to a small linear region, a $10-\mathrm{k} \Omega$ potentiometer combined with a following amplifier acted as an adjustable voltage divider to keep the signal within that portion. The potentiometer could therefore be adjusted such that the lower bound of the linear section was just above the noise floor, thus allowing only a current signal caused by particles striking the anode to be seen. The feedback resistors leading back to the HVPS were mounted on sockets so they could be easily adjusted for different expected currents.

In order to float the ammeter at high voltage, the circuit was entirely powered by $9-\mathrm{V}$ batteries. These voltage sources were passed through $+/-10-\mathrm{V}$ regulators and filtered by capacitors at both the input and output stages of the regulators. A 5-V regulator was used on the low-voltage side to drive the phototransistor. 


\section{Anode Deposition}

The deposition on the prototypes' anodes could be analyzed to determine beam characteristics. As particles being exhausted by the M-1 impact the anode, they adhere to its surface. The beam's divergence and uniformity could then be ascertained by observing the anode deposition.

The anode material needed to have surface properties such that the majority of the particles striking its surface were retained, thus reducing backsplash of particles that could interfere with those incoming. The anode needed to be electrically conductive such that minimal variation in the electric field existed between the anode and the accelerating gate. This condition meant that in vacuum, particle velocities should remain constant between the gate and the anode. Once the particles were collected on the anode, the deposition could be analyzed using both an optical microscope and a scanning electron microscope (SEM). The optical microscope provides a more global view of the anode deposition while the SEM allowed a close-up, detailed view providing insight on particle layering.

The M-1 prototype implemented mirror-finished stainless steel for the anode. The mirror-finish was chosen for the anode over the standard stainless steel used for the accelerating gate to ensure the surface was flat, uniform, and clean during prototype operation. Future prototype designs may consider applying a coating to the anode to improve particle adhesion.

\section{Automation Code and Data Acquisition}

Reduced gravity testing presents a unique environment for equipment operation and to the human body. To ensure data was achieved as consistently in flight as it was on the ground, experiment operation and data collection were streamlined using a LabVIEW automation program written by a ZESTT team member. The intent of this program was to minimize action needed by the flyers in case they became incapable of operating the experiment due to illness or other extenuating circumstances.

\section{Graphical User Interface Organization}

The LabVIEW graphical user interface (GUI), as seen in Figure 11, was designed for the flyers to be able to easily run the program during flight operations while permitting flexible parameter inputs.

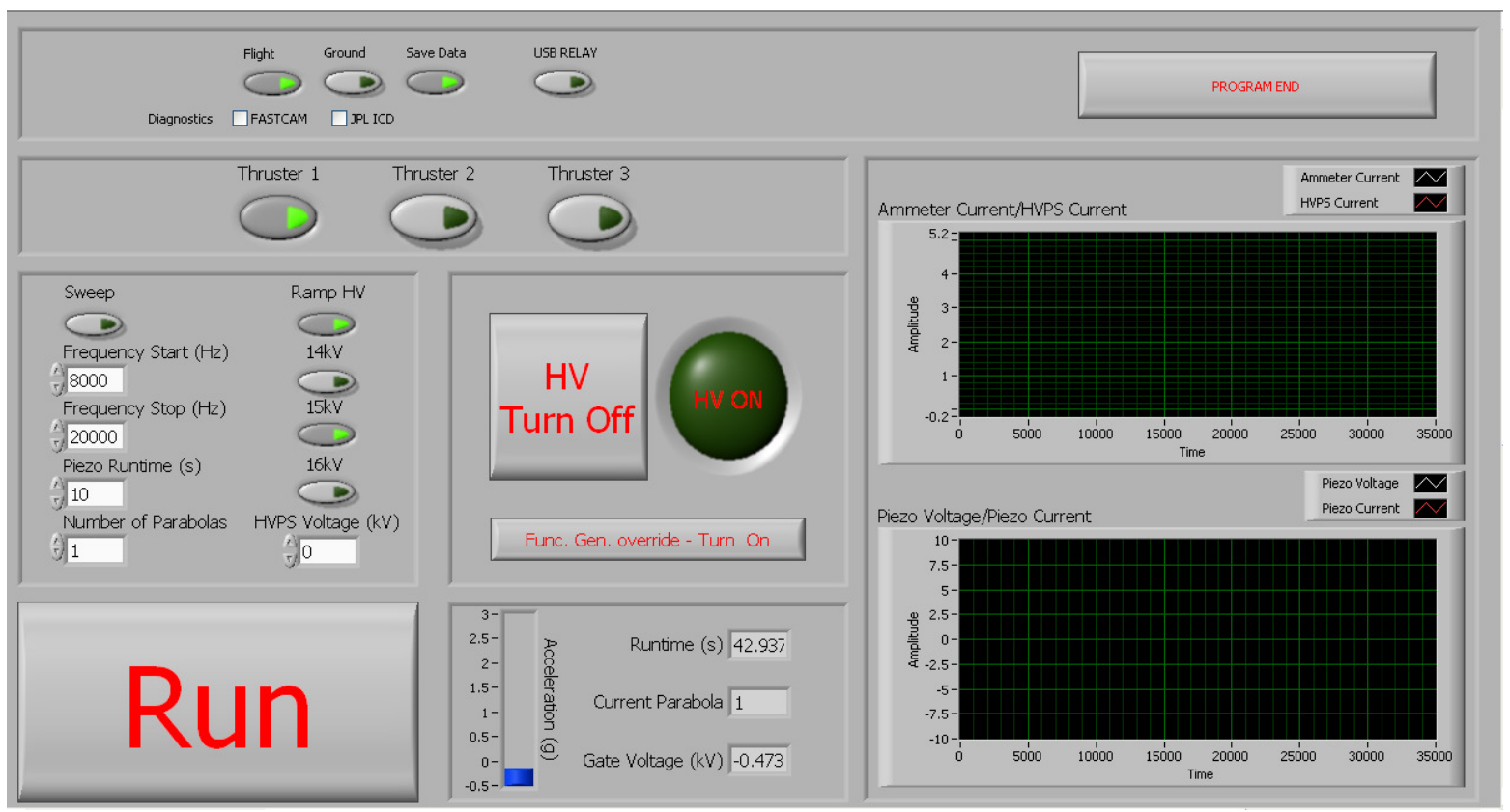

Figure 11. LabVIEW GUI for experiment automation. Both a flight and ground version of the GUI could be selected, thus allowing the user flexibility in varying the input parameters.

The top panel includes configurations for the major diagnostic tools used on the ground as well as in flight testing along with data save options that when selected would send a command to the DAQ to sample the desired channels. Indicator buttons for each M-1 prototype allows the user to know which prototype is being operated and, if needed, the ability to switch them. Parameters for activating the HVPS and function generator are displayed as default values for flight testing, but can be changed directly on the GUI with user input. 
A virtual LED is included to indicate when the high voltage is being recorded. Flight acceleration, runtime during the current microgravity parabola, and HVPS-sourced voltage are provided on the GUI to allow the flyers to monitor flight and M-1 conditions. During ground testing, no acceleration signal is present, and therefore the function generator override button (center of the GUI) allows the user to manually engage the piezoelectric. This override was also available during flight (but was never needed) had the aircraft's accelerometer signal been too noisy to provide proper triggering.

Plots of the data collected by the DAQ allowed flyers to monitor the ammeter and HVPS-supplied current as well as the piezoelectric voltage and current for the active M-1 prototype in real-time. The "Run" button initiates HVPS and function generator output as well as the data collection. Once pressed, this button reverts to a "Stop" button that serves as the automation master kill switch.

\section{Experiment Automation and Data Collection Scheme}

The experiment automation and data collection process controlled through the GUI is shown in Figure 12.

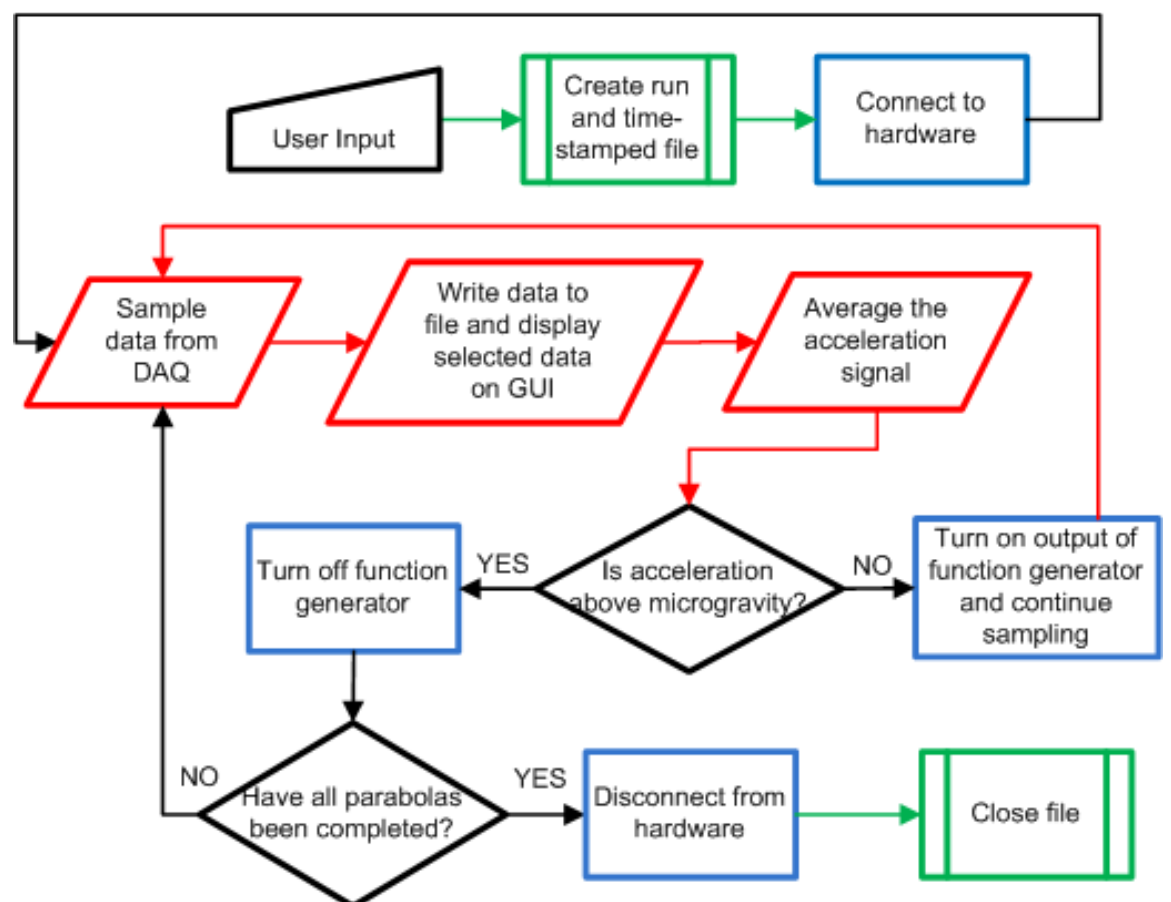

Figure 12. LabVIEW flight automation scheme. User input and decision points are indicated in black, internal LabVIEW processes in green, hardware interfacing in blue, and data collection in red. During ground tests, the function generator override button allows the operator to manually turn on/off the piezoelectric signal.

Once the LabVIEW program was started, the DAQ began to sample data, displaying it on the GUI and writing it to a time-stamped output file on the flight laptop. As soon as the acceleration fell into microgravity (below $0.05-\mathrm{g}$ ), LabVIEW commanded the function generator output to turn on. At the end of the microgravity period (above 0.1-g to account for pilot variation or noise on the signal), the output of the function generator turned off, and the parabola counter incremented. The saved file then closed, and a new file was created for data storage of the next parabola. For a different M-1 prototype to be tested, a manual switch engaging the prototype's electrical connections to the piezoelectric signal had to be flipped. Once the maximum number of parabolas was reached, LabVIEW disconnected the computer from the DAQ.

\section{Ground Testing}

Ground testing operations, primarily conducted by the science subsystem, began after proposal of the initial design configuration to better understand the behavior of the M-1 system and to build up the diagnostic tools. Theoretical predictions of the M-1 prototype's performance already existed; however, actual testing allowed the team to further refine their designs towards a configuration suitable for reduced gravity flight. Integrated systems testing incorporated lessons learned and procedures generated to familiarize all flight crew members with the experiment's operation. 


\section{A. M-1 Configuration Optimization Testing}

During the initial design phases, the team set out to investigate individual aspects of the M-1's design with the aim of optimizing the flight configuration through the lessons learned. The sieve, being an important interface influencing the prototype's performance, was tested and chosen carefully. Hole size, shape, and spacing along with the sieve material were all factors determining the particles' ability to pass through, charge, and emit individually. The team investigated woven and electroformed meshes, their differences shown in Figure 13.
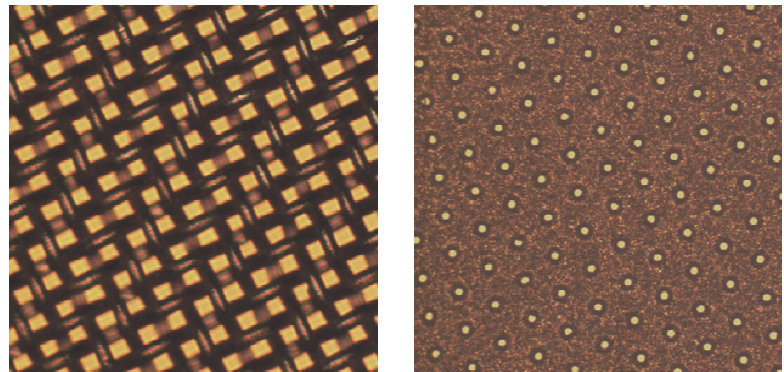

Figure 13. Sieve configurations. Microscope images of woven stainless steel mesh (left) and electroformed nickel mesh (right) at 100X magnification.

Preliminary tests with the woven sieve $(20-\mu \mathrm{m}$ holes) were conducted to determine if $1-15 \mu \mathrm{m}$ particles could pass through the holes when supplied vibrations from a piezoelectric. Following successful testing, the issue of sieve blinding and warping became the focus of investigation. After repeated operations, the sieve would become clogged or warped (distorting the holes) from the particles, necessitating the use of compressed air and ultrasonic baths to dislodge them.

The electroformed sieve $(10-\mu \mathrm{m}$ holes $)$ was chosen to overcome the warping issues associated with the woven sieve. A tighter particle range of 1$10 \mu \mathrm{m}$ was used with the electroformed sieve, thus providing a closer fit to enforce single particle emission. Post-test analysis showed only a handful of particles now became lodged within the sieve holes; however, blinding effects were still observed, preventing consistent particle emission.

The team systematically stepped through the possible causes of this effect and tested various M-1 configurations to eventually determine humidity to be the primary problem. As the particles were exposed to the atmosphere, water vapor in the air adhered to them, causing significant particle clumping and packing beneath the sieve. Knowing the current test environment was only a fraction of the relative humidity that would be present during flight testing in Houston, a particle drying technique was implemented. Particles were first heated for several minutes in a closed glass vial using a heat gun. The vial was then opened and placed in a sealed plastic bag filled partially with a silica gel desiccant to remove the bulk of the moisture. Once the particles were dried (when no clumping was observed during handling), they were quickly loaded into the M-1 and sealed in the vacuum chamber for testing.

In addition to removing moisture from the system, previous studies have shown that introducing oversized particles to a particle reservoir improves sieving efficiency. ${ }^{9}$ The team therefore decided to apply this concept to the M-1 prototype. A layer of oversized particles (silverized soda lime glass) on the order of $53-\mu \mathrm{m}$ or $200-\mu \mathrm{m}$ were added between the sieve surface and the $1-10 \mu \mathrm{m}$ particles contained in the reservoir as shown in Figure 14. The piezoelectric vibrations caused the oversized particles to break up clumps of smaller particles, thus allowing them to pass through the sieve. As particles larger than 200- $\mu \mathrm{m}$ were applied, they became too heavy to be moved effectively by the piezoelectric. The 53- $\mu \mathrm{m}$ particles were found to have the greatest effect on emission and were therefore used in the flight configuration. These methods combined were shown to improve emission significantly as a steady stream of particles was now produced unlike the handful of particles emitted previously.

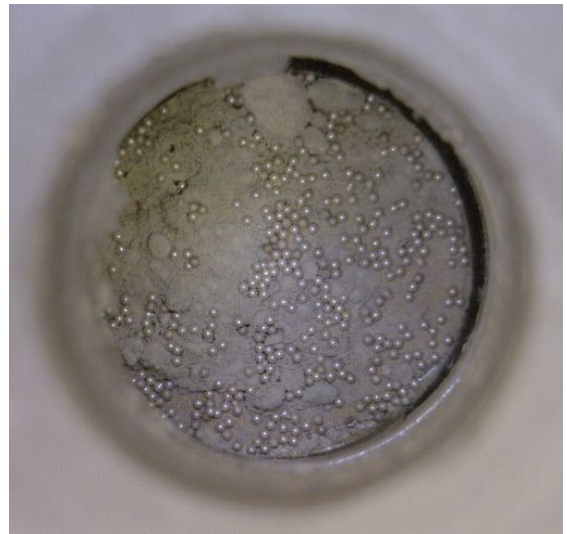

Figure 14. Oversized particles contained within the particle reservoir. 200- $\mu \mathrm{m}$ oversized particles were used with 1-10 $\mu$ m silver-coated soda lime glass particles.

\section{B. Integrated System Testing}

Once the flight configuration had been determined, the M-1 prototype was integrated with the flight experiment setup (thruster testbed). During the month preceding the reduced gravity flights, integrated system tests were conducted to fully prove out the experiment setup and to determine the M-1 operating conditions to be tested in microgravity. Flight crew members were given the opportunity to conduct these tests to gain understanding and experience with all experiment systems. While each member had in-depth knowledge of a few systems, each flyer needed to be capable of operating the entire experiment and troubleshooting problems during flight. 
Determining the optimal M-1 operating parameters required the use of the various diagnostics described in Section III.C. The particle tracking velocimetry diagnostic was used primarily during testing to visually verify particles were being emitted and to qualitatively determine the optimal test conditions by observing the throughput density as shown in Figure 15. Due to the laser power available to the team, individual spheres during vacuum tests could not be captured to allow quantitative measurements using the post-processing program.

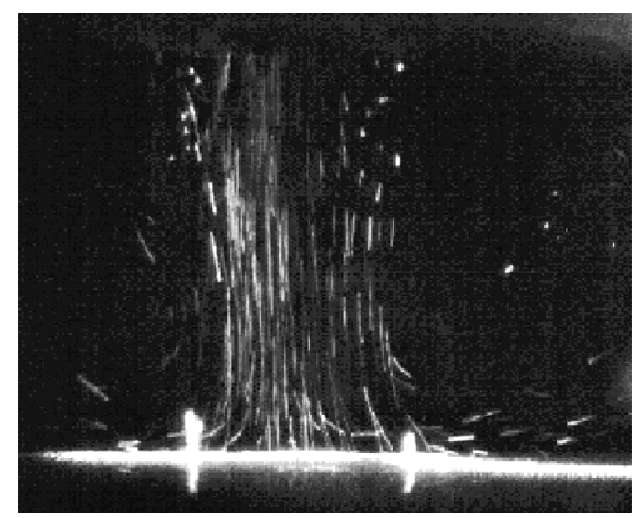

Figure 15. PTV test results. Particles tested in atmosphere with an electric field set at $1.5 \times 10^{6} \mathrm{~V} / \mathrm{m}$ and frequency at $7 \mathrm{kHz}$.

The piezoelectric frequency, amplitude, and applied acceleration as well as the applied electric field were the main operating variables to be decided. To validate the hypothesis that piezoelectrics would improve emission, the M-1 was first tested varying only the electric field with no applied vibration. No emission was observed. Immediately when vibration was applied, a burst of particles were emitted.

Once it was determined that vibration was indeed necessary for emission, various combinations of piezoelectric frequency, amplitude (combined with frequency to provide various accelerations), and electric fields were applied to determine when emission was optimal. From testing, it was evident that increasing both amplitude and frequency produced the largest emission as the acceleration grew. Due to limitations of the amplifier, it was found that the emission was best when the frequency reached the amplifier's resonant points $\left(8\right.$ and $11 \mathrm{kHz}$ at $\left.100 \mathrm{~V}_{\mathrm{ppk}}\right)$ where the piezoelectric provided the most displacement.

The applied electric field was not the driving condition in the M-1 prototype's operational parameters. As long as the voltage was well above the particle lift-off threshold, the M-1 emitted. The electric field was therefore set at $1.5 \times 10^{6} \mathrm{~V} / \mathrm{m}$ for all subsequent tests, which was typically slightly below the breakdown potential of air. Due to the fact each M-1 prototype was fabricated by students, some tuning was needed to ensure optimized conditions.

The ammeter was also adjusted during ground testing to provide current readings of the particles striking the anode. Preliminary data taken with the ammeter during bench-top testing suggested that particle emission could cause the entire signal plus noise to go beyond the transistor saturation point before decaying within the saturation bounds. As testing began, noise became a major limiting factor in the robustness of the ammeter. When the ammeter was floated on top of high voltage, the gain, because of noise issues, could not be raised to 1000X, which is what was needed to measure on the nano-amp scale. Determining the sources of the noise, as a result, became a primary task. The HVPS and piezoelectric amplifier were major noise producers, and the current was seen to fluctuate when the equipment was operated. One possible noise source was inductive coupling due to the large (1-2 amp peak-to-peak) oscillations in the piezoelectric lines and capacitive coupling between the piezoelectric and the grounded sieve. Another possible noise source was the micron vibrations of the sieve causing oscillations in the capacitance and electric field between the sieve and gate.

The electrical/CDH team went to great effort to decrease noise by wire wrapping the piezoelectric lines to reduce inductive coupling and grounding everything directly to the vacuum chamber. While this had significant results in reducing piezoelectric noise, noise due to the HVPS and environment (i.e. lights, personnel, etc.) were yet to be reduced. Further investigation would be needed to fully understand and overcome the ammeter's noise limitations.

While the ammeter was the main in-flight means of gathering M-1 performance information, one final method of understanding the emission characteristics was the deposition left on the anode, which provides information on emission uniformity and beam divergence. Throughout ground testing, the anode was examined visually through the use of an optical microscope. When the $53-\mu \mathrm{m}$ oversized particles were used with the 1-10 $\mu \mathrm{m}$ propellant particles, the deposition tended to have a densely focused center
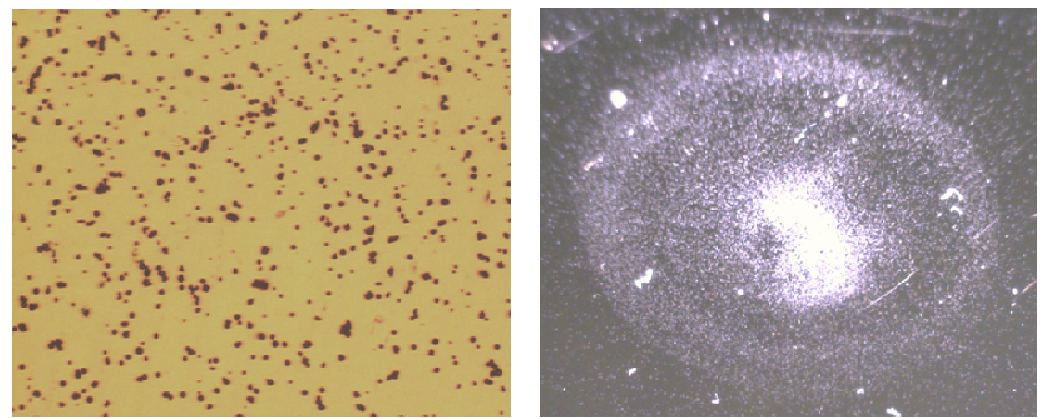

Figure 16. Microscope images of anode deposition. Electroformed sieve allows single particle emission (left). 53-um oversized particles produced a concentrated beam center (right).

14

American Institute of Aeronautics and Astronautics 
spreading out in rings as seen in Figure 16. With the $200-\mu \mathrm{m}$ oversized particles in the reservoir, the deposition spread out in a much wider, even layer having no concentrated center. Both configurations were tested under the same conditions leading the difference to be based on how the piezoelectric induces movement of the oversized particles.

\section{Reduced Gravity Operations}

Following ground testing, the team participated in NASA's Reduced Gravity Student Flight Opportunities Program in Houston, Texas in June 2009. The program provided two flight opportunities to test the NanoFET prototypes onboard a Boeing 727 aircraft that generates microgravity test environments through parabolic flight profiles over the Gulf of Mexico. Using the lessons learned from ground tests, the flight configuration and operating parameters were implemented.

\section{A. Flight Environment}

Parabolic flight introduces a unique testing environment for both experimentation and the experiment operator. Each flight consisted of 30 zero-g parabolas, one lunar gravity parabola (onesixth Earth's gravity), and one Martian gravity parabola (one-third Earth's gravity). During a single parabola, 18-25 seconds of microgravity was provided along with an associated high-gravitational period of approximately 1.8 -g. ${ }^{10} \quad$ As indicated in Figure 17, an entire parabola sequence took approximately 65 seconds to complete. After 17 parabolas, a turnaround period of 35 minutes occurred where the plane was in

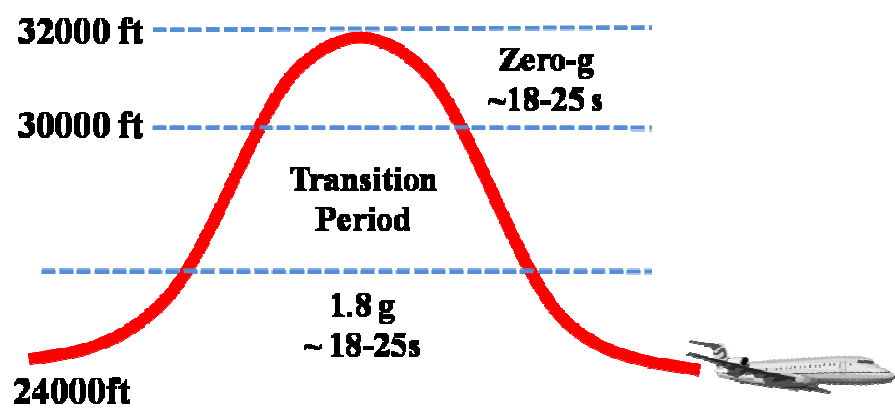

Figure 17. Parabolic flight profile. Two flight days of 32 parabolas each were provided. One parabola lasted approximately 65 seconds. steady flight as it reversed direction back towards Houston, TX before parabolic flight resumed. The entire flight from take-off until touchdown at Ellington Field, where the flights were based, lasted for approximately 1.5 hours.

For ZESTT, the zero-g period was the primary focus for testing, however the 1.8-g and transition periods needed to be considered when designing the experiment. Equipment such as the pressure gauge and turbo pump along with the M-1 prototypes were oriented such that the changes in gravitational loading would not alter their operation. The aircraft was manually piloted and therefore produced slight variations in the gravitational levels achieved and the length of each parabola flown. The LabVIEW program was designed to activate the M-1 prototype for a 10-second

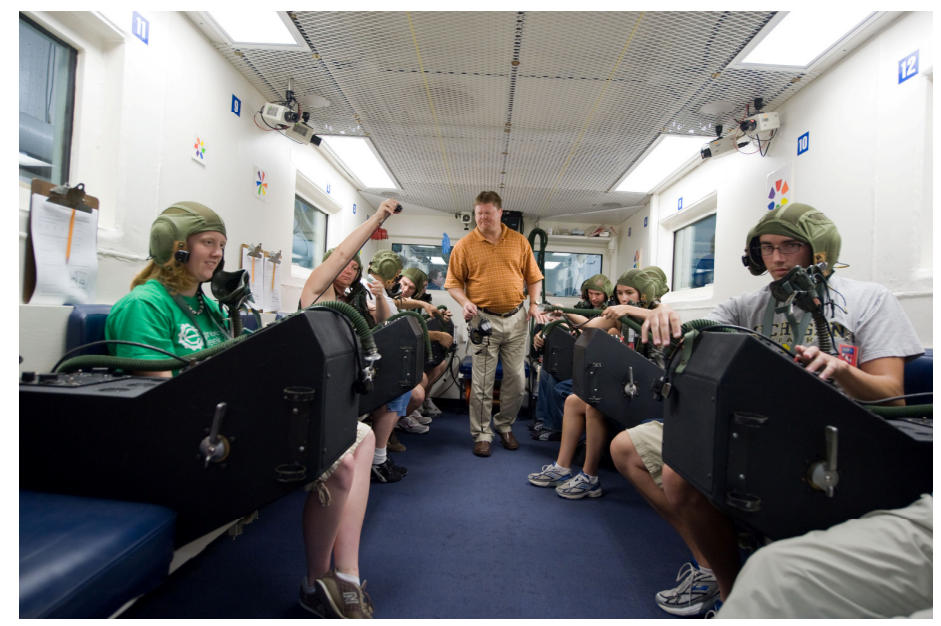

Figure 18. Hypobaric chamber training. Pressure within the chamber was reduced to simulate 25,000-ft altitude, thus allowing students to identify their symptoms of hypoxia and to take corrective action. window during the zero-g period to ensure consistency in each test (accounting for shorter parabolas). Acceleration data was recorded for each parabola to understand the conditions of individual tests.

The microgravity flight environment can be taxing on the human body and affects all flyers differently. In preparation for microgravity testing, the flight crew underwent classroom and hypobaric chamber training as shown in Figure 18. These sessions, taught by NASA officials, introduced the flyers to the various biological effects (e.g. hypoxia, decompression and motion sickness, spatial disorientation, etc.) that they might experience during flight. They were first taught preventive measures but also how to recognize their symptoms and take corrective action if needed. Hypobaric 
chamber training allowed the flyers to experience first-hand their symptoms of hypoxia in a controlled setting. Each crew member was put on oxygen, and the pressure inside the chamber was reduced to simulate 25,000 -ft altitude. The students were then instructed to remove their oxygen masks until they recognized they were hypoxic or the allotted time expired. While cabin pressure loss was not anticipated during flight, the crew was adequately prepared for the test environment.

Prior to flight, flyers were also given medication (SCOP-DEX) to aid in motion sickness prevention. The environment was entirely new to all flyers, and their bodies were therefore not used to the lack of gravity or additional loading experienced during the high-g periods. Thus, manipulating their fingers and bodies became more difficult. Time for recording observations and switching M-1 prototypes was built into the flight plan such that minimal operation was encouraged during the 1.8 -g period.

In order to ensure the flyers were adequately prepared for any incident during flight, a two-pronged approach was taken to plan contingencies. During the first stage, all team members developed a series of possible problems that could occur, the likely cause of the malfunction, and the best solution within their systems of expertise. Once the list of contingencies was compiled, the flyers were required to complete a quiz over all experiment's systems to be sure they were ready to fly. In the quiz, the same sort of information was requested; the malfunction was listed, and the flyers were asked to correctly identify the likely cause of the problem and how it could be solved.

\section{B. Flight Experience and Outcome}

Reduced gravity flight is a rare opportunity and rewarding experience. Flight crew members of the ZESTT team were not only able to experience weightlessness within the bounds of gravity, but they also flew and tested an experiment that they designed and built themselves. While technological strides had been taken throughout the year to design, build, and test the NanoFET M-1 prototype and testbed system, the microgravity flight was an opportunity to better understand its operations in a more relevant "space-like" environment.

The flight environment is an incredibly challenging atmosphere. The ZESTT team was able to successfully demonstrate a fully automated thruster testbed, via LabVIEW, capable of supporting future experiments. The LabVIEW automation scheme worked flawlessly allowing the flyers to focus on monitoring the systems and troubleshooting arcing that occurred within the M-1 prototypes.

On both days, the flyers were able to stabilize the voltage to the desired level of $15 \mathrm{kV}$ prior to parabolic flight. As soon as parabolic flight began, intermittent arcing was seen occurring mainly between the electrodes within the prototype, but also around the acceleration gate's connection. This arcing primarily occurred at the beginning and end of every parabola during the transition periods and was most likely due to material that had not fully outgassed, thus increasing the local pressure. The flyers would frequently need to adjust the voltage until the prototype stabilized. After approximately half of the parabolas were completed, the intermittent arcs stopped, requiring no further voltage adjustments to be made.

Despite efforts during ground testing to remove the effects of humidity in M-1 emission, the high humidity levels in Houston still posed a problem. Exposure for even a brief amount of time caused the moisture from the air to bind the particles together, thereby requiring significantly higher, unattainable vibration and electric fields for particle lift-off to occur. Due to the inability to pump down the vacuum chamber overnight while installed on the aircraft, only about four hours of total pumpdown time was achieved prior to each flight test. This reduced pumpdown time for water vapor outgassing contributed to particle adhesion issues that impeded consistent particle emission from the M1 prototypes during flight. Finally, electrical noise was a significant issue on the aircraft and prevented the ammeter's ability to capture the nano-amps of current transferring from the particles to the anode. Under less noisy conditions, the ammeter line could be magnified to show a change in current when particles struck the anode; however, the signal-to-noise ratio in the aircraft was too high for the ZESTT flight configuration.

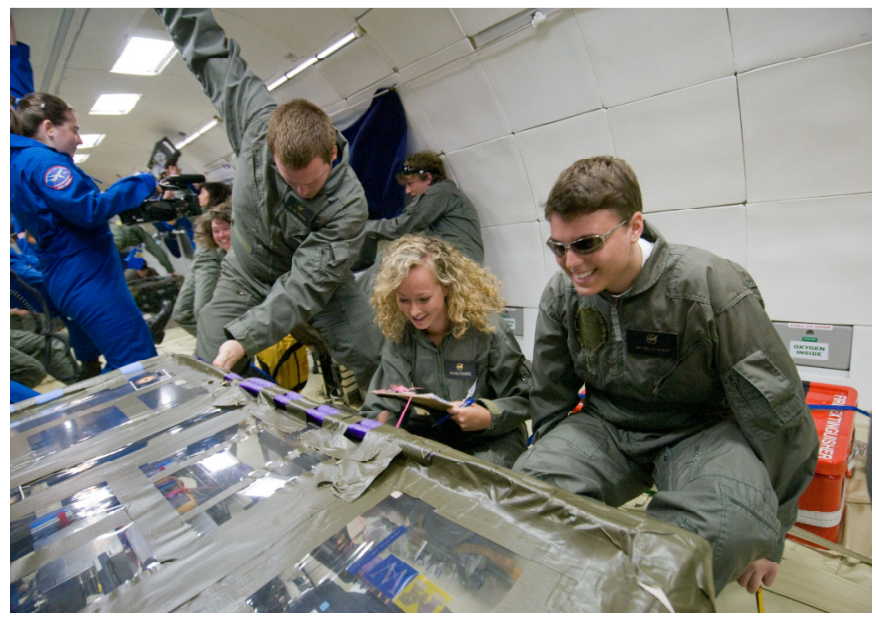

Figure 19. Reduced gravity flight testing. University of Michigan students ran 26 tests each day of the M-1 prototypes.

16

American Institute of Aeronautics and Astronautics 
While the team put forth a strong effort to plan for contingencies, the issues that occurred during flight testing limited the useable experimental data that could be gathered. The lessons learned through the experience, however, have provided greater insight into flight operations and will be implemented in future NanoFET designs. Conducting an experiment in reduced gravity provided each flyer with a unique flight experience that they will carry with them into their professional careers.

\section{ZESTT Project Benefits}

While technology development drove the ZESTT project, the educational experience was also important. The technical and personal lessons learned were unique to each individual and significantly contributed to their academic development. Transferring their engineering drive and experience, ZESTT students sought to inspire younger students to enter STEM (science, technology, engineering, and mathematics) fields.

\section{Educational Benefits}

Student development was an important aspect of the ZESTT project. The project's aim was to enhance the lessons that all team members have learned from their formal classroom training by providing a hands-on, fast-paced experience with real-world engineering. In addition, each student was exposed to various fields (e.g. vacuum systems, high voltage, electric propulsion, etc.) not commonly taught in the undergraduate curriculum.

Working on ZESTT was not only a great experience for students to take part in working on something new and exciting, but it also provided them with real, first-hand engineering experience. For many students, this was their first in-depth exposure to all stages of the design-build-test-fly cycle, and for some students, this was their first engineering team. Those with prior experience took on leadership positions. These students learned the importance of task delegation, decision making, conflict resolution, and mentoring their fellow team members to bring them to a level where they could complete the job. Younger team members focused on specific areas to develop their technical skills as they experienced the full project cycle.

Teamwork and communication were some of the most important skills ZESTT members gained over the course of the year. While sometimes frustrating, constant design modifications were necessary to produce a well-refined product compliant with all requirements and objectives. Once the design was created, team members had to learn how to fabricate it, often incorporating modifications as they understood their own fabrication capabilities. Team members involved in testing discovered needed improvements for each design iteration. Communicating specifically what needed to be done was critical in preparation for flight. Students learned how to be flexible and quickly adjust when problems or changes arose.

The benefits of the ZESTT project are numerous and extend far beyond those of a typical extra-curricular activity. The complex project provided a challenging atmosphere for student growth. ZESTT placed high demands on each individual as they worked to meet deadlines while taking classes full-time. The lessons learned and skills gained, however, provided these undergraduate students with a solid foundation and introduction to the professional world.

\section{Outreach}

In addition to scientific research, the ZESTT team led an ambitious community outreach program made possible by the Michigan Space Grant Consortium. ${ }^{11}$ The team conducted workshops and assemblies that focused on gaining interest in the STEM fields among elementary and middle school students through rocket and glider building, wind
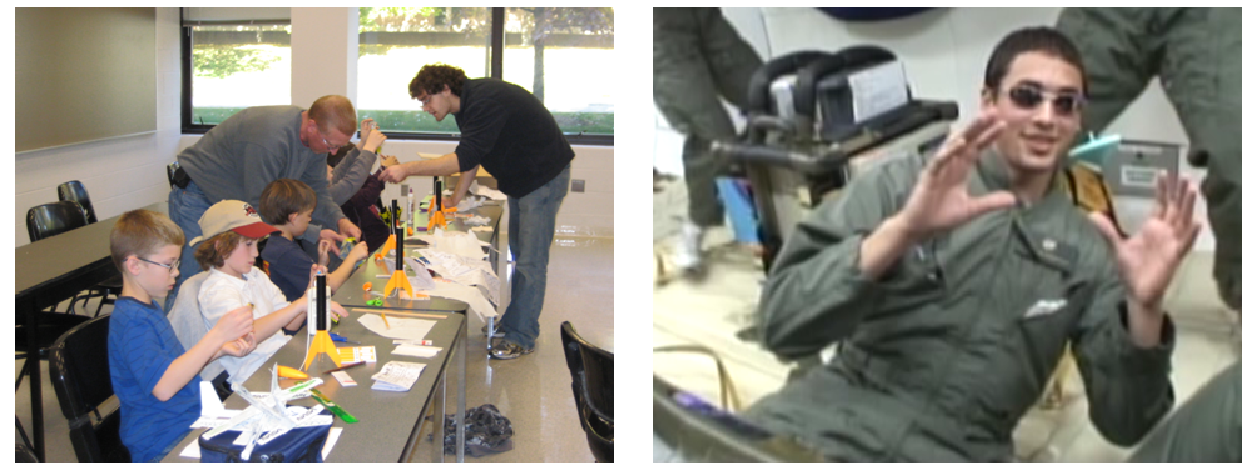

Figure 20. Outreach initiatives. $K-12$ students learn to build model rockets during a workshop (left). A flight crew member demonstrates the spinning motion of a top in microgravity (right). 
tunnel tours, and other activities. The workshops were expanded upon those previously given to include more multidisciplinary engineering and teambuilding activities. Assemblies were also conducted for grade-school children. These assemblies consisted of three main components: a video produced by the team showing past experiments and talking about microgravity, a discussion about engineering and sciences, and a few interactive and educational games in which students could take part. Each assembly was tuned to the needs of the school and location giving it a life of its own.

This year, the team began working with a local museum to create an interactive hands-on exhibit to expand the audience that could be reached. The exhibit would provide both students and their parents with a unique experience that teaches them fundamentals about space. Due to time and resources, the exhibit was not produced; however, the fundamental research and designs could be used in the coming years to complete the project.

A major portion of the team's outreach initiatives was a secondary outreach competition, which gave K-12 students the opportunity to design their own mini-experiment to be flown in microgravity. The team dedicated two parabolas to fly the chosen experiments. The nature of this experiment, to showcase interesting behavior in microgravity, was proposed by the students who attended the workshops and assemblies. The chosen ideas included demonstrating the gyroscopic motion of a spinning top and the precession of a yo-yo in the weightless environment. Both experiments were videotaped to be shared with the students.

\section{Conclusion}

The ZESTT project has successfully combined technical research with education to positively impact the future, laying the groundwork for future teams to come. Students involved in the project were able to fully experience a design-build-test-fly cycle fulfilling S3FL's objectives and further instilling the skills learned within the classroom. While quantitative data was not obtained during either reduced gravity flight, a vacuum testbed was implemented with proven automation providing the means for sustaining future microgravity flights. The lessons learned can be applied as NanoFET continues to develop.

The team accepted a challenging set of objectives at the start of the year. Not only was the first full operational NanoFET prototype containing a piezoelectric feed system developed, but the diagnostic tools to be able to analyze its performance were also created. Great strides have been taken to understand the dynamics of the M-1 prototype in an effort to validate NanoFET performance models. Extensive ground testing prior to flight aided in the optimization of the M-1 design. The changes throughout the year were well documented and tracked to ensure improvements were made in the next NanoFET iteration.

Student growth and outreach were essential within the educational environment introduced through involvement with S3FL. The ZESTT team successfully completed all educational objectives to ensure the team's growth was stimulated and progress was tracked. Team members developed a wide variety of skills throughout the year. Some gained leadership skills learning how to manage subsystem teams, while others focused more on improving their technical abilities such as learning software packages including SolidWorks, MATLAB, and COMSOL Multiphysics.

Managing the team was aided through the use of subsystems (structures/mechanisms, science, electrical/CDH, business, and outreach). Each subsystem had their own set of objectives allowing individuals to focus closely on specific aspects while the leads from each group formed the systems/operations team to integrate the team's work. Structures/mechanisms team members learned how to first design the M-1 prototype, modeling and verifying the integrity of their designs before fabricating it. Those on the science team learned how to systematically test a design handed to them by another subsystem as well as to conduct some of the fundamental research that supported the science behind the initial design. NanoFET challenged the electrical/CDH subsystem to expand their knowledge of circuitry and programming.

The business and outreach teams were comprised of a collection of students from each of the three beforementioned subsystems. These members were not only challenged technically, but they also learned how to interact with potential sponsors and K-12 students. Each team members participated in the outreach activities providing "beyond the formal classroom learning" opportunities that opened the eyes of many K-12 students to engineering and technology.

All team members learned the importance of communication. Meetings between the subsystem leads and management provided a forum for everyone to learn about various aspects of the other systems. In addition, an online worksite provided the means for compiling procedures and lessons learned for all members to see. Design reviews primarily set the milestones the team worked towards as they prepared for microgravity testing. Each review allowed the students to communicate with professionals on what they had done and to receive feedback on their designs. 
Using the knowledge acquired through the ZESTT project and the work done over the course of the year, NanoFET will continue to move forward. The M-1 design will continue to be improved upon as the NanoFET prototypes move into the nanometer-sized particle regime. ZESTT has implemented a testbed in which new NanoFET prototype designs can be investigated in the unique microgravity environment made available through NASA's Reduced Gravity Student Flight Opportunities Program. The experiences the students have gained as being part of this project will carry with them in their future endeavors.

\section{Acknowledgments}

The ZESTT project was financially supported by the Michigan Space Grant Consortium (MSGC), Women in Science and Engineering (WISE), and the University of Michigan (UM) College of Engineering. Special thanks go to the ZESTT project team members including Theresa Biehle, Benjamin Brierty, Nicholas Ciarelli, Alexandra Doan, Michelle Knapp, Phil Le, Huy Nguyen, Michael Ramirez, and Yuly Wung who made this project possible. In addition, thanks go to Mark Stakhiv and Jeffrey Schloemer who were instrumental in the PTV diagnostic development. ZESTT would like to thank NASA's Reduced Gravity Office staff for providing the two microgravity flight test opportunities and their help throughout the preparation process. Additional thanks go to the UM Student Space Systems Fabrication Laboratory (S3FL) and the Plasmadynamics and Electric Propulsion Laboratory (PEPL) for providing resources and support. The Space Physics Research Laboratory (SPRL), specifically Robb Gillespie and Ken Arnett, provided valuable technical advice and resources. Finally, thanks go to the UM Aerospace Engineering technical staff (Thomas Griffin and David McLean), Professor Peter Washabaugh (Aerospace), and Michael Lee with the Wilson Student Project Center who were all especially instrumental during the experiment's fabrication.

\section{References}

${ }^{1}$ Liu, T., Musinski, L., Patel, P., Gallimore, A., Gilchrist, B., and Keidar, M., "Nanoparticle Electric Propulsion for Space Exploration," in Space Technology and Applications International Forum - STAIF 2007, edited by M. S. El-Genk, American Institute of Physics, Albuquerque, NM, 2007, pp. 787-94.

${ }^{2}$ Morris, S., Drenkow, B., Liu, T., Biehle, T., Munski, J., Han, H., Swieringa, K., Martinchek, P., Knapp, M., Trabert, R., Wind, R., Brierty, B., Gilchrist, B., and Gallimore, A., "Reduced Gravity Testing of the Nanoparticle Field Extraction Thruster Concept," AIAA-2008-4995, 44th AIAA/ASME/SAE/ASEE Joint Propulsion Conference, Hartford, CT, 21-23 July 2008.

${ }^{3}$ Liu, T., Deline, C., Ramos, R., Sandoval, S., Smetana, A., Gilchrist, B., Washabaugh, P., and Renno, N., "The Student Space Systems Fabrication Laboratory: An Approach to Space Systems Engineering Education," ASEE2006-1917, 113th ASEE Conference, Chicago, IL, 18-21 June 2006.

${ }^{4}$ Mankins, John. "Technology Readiness Levels: A White Paper", Office of Space Access and Technology, NASA, 1995.

${ }^{5}$ Liu, T., Drenkow, B., Musinski, L., Gallimore, A., Gilchrist, B., Mirecki-Millunchick, J., Morris, D., Doan, A., Munski, J., and Muldoon, A., "Developmental Progress of the Nanoparticle Field Extraction Thruster," AIAA-20085096, 44th AIAA/ASME/SAE/ASEE Joint Propulsion Conference, Hartford, CT, 21-23 July 2008.

${ }^{6}$ Musinski, L., Liu, T., Eu, I., Gilchrist, B., Gallimore, A., Millunchick, J., and Morris, D., "Nanoparticle Field Extraction Thruster (NanoFET): Introduction to, Analysis of, and Experimental Results from the 'No Liquid' Configuration," AIAA-2008-5097, 44th AIAA/ASME/SAE/ASEE Joint Propulsion Conference, Hartford, CT, 2123 July 2008.

${ }^{7}$ Hein, K., Hucke, T., Stintz, M., and Ripperger, S., “Analysis of Adhesion Forces Between Particles and Walls Based on the Vibration Method," Part. Part. Syst. Charact., (2002).

${ }^{8}$ Devasenathipathy, S., Santiago, J.G., Wereley, S.T., Meinhart, C.D., Takehara, K., "Particle imaging techniques for microfabricated fluidic systems," Experiments in Fluids, 34 (2003), pp. 504-514.

${ }^{9}$ Liu, K., "Some factors effecting sieving performance and efficiency," Powder Technology, 193 (2009), pp. 208-213.

${ }^{10}$ Sopensky, E., “Trying out zero gravity,” Potentials, IEEE, Vol.17, No.3, pp.38-41, Aug/Sep 1998.

${ }^{11}$ Liu, T., and Richards, B., "Saturday Aerospace Workshops," Michigan in Space, Vol. 8, September 2005, pp. 15-16. 